\title{
MedienPädagogik
}

Zeitschrift für Theorie und Praxis der Medienbildung

Themenheft Nr. 31: ‘Digitale Bildung〉

Medienbezogene Bildungskonzepte für die «nächste Gesellschaft).

Hrsg. v. Jasmin Bastian, Tobias Feldhoff, Marius Harring und Klaus Rummler.

\section{FELS - Forschend-Entdeckendes Lernen mit dem Smartphone}

Jirka Müller, Uta Magdans und Andreas Borowski

\begin{abstract}
Zusammenfassung
Obwohl die Schulleistungen der Schülerinnen und Schüler in Physik seit dem Pisa-Schock zunahmen, gehört Physik nach wie vor zu den unbeliebtesten Fächern im Schulkanon. Gerade einmal 15,3\% wollen einen naturwissenschaftlichen oder ingenieurstechnischen Beruf ergreifen, 9,2\% unterhalb des OECD-Durchschnitts (PISA 2015). Um dem entgegen zu wirken, werden neue motivations- und interessenssteigernde Methoden gebraucht. Der von uns entwickelte Lernzyklus FELS (Forschend-Entdeckendes Lernen mit dem Smartphone) stellt eine solche Methode dar. Ziel ist es, die Sinnhaftigkeit von Physikunterricht in den Augen der Schülerinnen und Schüler zu erhöhen und so affektive Aspekte zu steigern. Dies soll durch einen Anschluss an die Erfahrungswelt der Lernenden mit Hilfe smartphonebasierter Experimente innerhalb eines forschend-entdeckenden Unterrichts ermöglicht werden. Das forschend-entdeckende Lernen soll die unterrichtliche Einbettung gewährleisten. Der lebensweltliche Anschluss kommt dadurch zu Stande, dass die Schülerinnen und Schüler ausserschulisch innerhalb ihrer Erfahrungswelt in Form von Hausaufgaben innerhalb eines blended-learning Szenarios experimentieren. Ein Vergleich mit den Standards der KMK bezüglich Medienkompetenz (KMK 2016) zeigt, dass FELS ein probates Mittel darstellen kann, um die entsprechenden Rahmenkompetenzen in den Physikunterricht einzubringen.
\end{abstract}

FELS - Inquiry-based-learning with the smartphone

\begin{abstract}
Although student achievements increased in physics education since the shock caused by the first PISA test, physics education is still one of the most unpopular subjects in school. Only 15,3\% of the students, 9,2\% below the OECD country average, intend to persue a science-oriented career or a career in engineering (PISA 2015). New methods are needed to improve the students' motivation and interest in becoming scientists and/or engineers. We developed FELS (Forschend-Entdeckendes Lernen mit dem Smartphone - InquiryBased Learning with the Smartphone) as a method to engage students affectively and to create more meaningful physics lessons for students. Both aims are achieved by using students' smartphones to connect their daily life with scientific experiments in an inquiry-
\end{abstract}


based learning scenario. By experimenting outside of school using homework in a blendedlearning scenario, a direct link to the students' everyday life is made. A comparison with the national standards of the KMK dealing with (new) media shows that FELS can be an appropriate tool to introduce the relevant competencies into physics lessons.

\section{Motivation}

Nach wie vor ist Physik ein unbeliebtes Schulfach (PISA 2015). Ein möglicher Grund hierfür sind fehlende lebensweltliche Anschlüsse bei Schülerexperimenten. Um den Physikunterricht (PU) künftig interessanter zu gestalten, gilt es stärker als bisher die Art des PUs so zu ändern, dass Experimente an die Lebenswelt der Jugendlichen anknüpfen.

Das Ersetzen alter Taschenrechner oder verstaubter Messinstrumente mit dem im Alltag der Jugendlichen vielgenutzten und beliebten Smartphone (JIM-Studie 2016) würde den PU nur scheinbar aufwerten. Stattdessen bedarf es einer Neukonzeptualisierung des PUs, die den didaktischen Möglichkeiten des Smartphones gerecht wird (vgl. Spang 2014) und einen stärkeren Bezug zur Erfahrungswelt der Lernenden hat.

Für einen besseren Anschluss an die Lebenswelt der Jugendlichen muss zunächst geschaut werden, wie diese aussieht. Innerhalb des letzten Jahrzehnts hat moderne Technik das Leben nicht nur geprägt, sondern auch verändert. Als prominentes Beispiel dient gemäss der Einschätzung von O'Reilly (2006) das Web 2.0, welches er als "the business revolution in the computer industry» bezeichnet. Internetinhalte konnten nun auch vom normalen Endverbraucher erstellt werden, wodurch die Nutzungsmöglichkeiten des Internets anstiegen. Wenn dieser Umbruch bereits als Revolution (ebd.) bezeichnet wird, um wie viel mehr stellt es erst die Möglichkeit dar, mit einem Smartphone multimediale Inhalte an jedem Ort zu jeder Zeit zu generieren und auf eigenen Seiten zu veröffentlichen. Mit einer Verbreitung von über $90 \%$ bewirkte das Smartphone als mobiles Endgerät eine ähnliche Revolution im Alltagsleben heutiger Jugendlicher im Alter von 12 bis 19 Jahren (JIM-Studie 2015, 46), wie damals die Einführung des web 2.0.

Bisher kleideten Textaufgaben die Fachproblematik in Kontexte ein, die diese Verhaltens- und Erfahrungsänderungen nur wenig berücksichtigten. Im Projekt "Schulkontext» (Oberländer 2014) werden solche Änderungen berücksichtigt. Bei "Schulkontext» halten alltägliche Geräte und Medien Einzug in den Unterricht, um den angestrebten Lebensweltbezug von Schule zu generieren. Konkret auf den PU bezogen bedeutet ein solcher Einzug, dass Smartphones als Messinstrument (u.a. Vogt et al. 2011; Lück und Wilhelm 2011) und Dokumentationshilfe (Rath und Schittelkopf 2011) bei Experimenten genutzt werden, was Kuhn, Vogt und Müller (2011) in Anlehnung an das situierte Lernen als «materielle Situiertheit» bezeichnen. Zeitgleich erhöht die Nutzung eines vertrauten Alltagsgegenstandes die Authentizität 
von PU, weil naturwissenschaftliche Kenntnisse auf Grundlage der Erfahrungswelt der Schülerinnen und Schüler konstruiert werden (Höttecke und Rieß 2015).

Die Art der Lernumgebung entscheidet über die unterrichtliche Einbettung, die u.a. nach Tesch und Duit (2004) ein wichtiges Qualitätsmerkmal eines guten Unterrichtsverlaufs darstellt. Horvath $(2007,36)$ spricht sich gegen eine zwischen Lernenden und Lehrenden entfremdende «Bewahrpädagogik» aus und plädiert für eine Umgestaltung der Lernsituationen. Anstatt sich von der Lebenswelt der Lernenden im Unterricht zu distanzieren, soll vielmehr die Kreativität und Dynamik der ausserunterrichtlichen Mediennutzung der Jugendlichen unterrichtlich genutzt werden. Dies umfasst insbesondere die Nutzung des jugendlichen Gestaltungswillens der Jugendlichen und das zugehörige Interaktionspotential mobiler Endgeräte sowie entsprechende Medienbildung im weiteren Sinne. Insofern erscheint es bei der Einführung neuer Experimentiermittel wie dem Smartphone ratsam, neue Lernumgebungen zu entwickeln und eben nicht auf alte und bestehende zurückzugreifen.

Die Verfügbarkeit von Smartphones sollte nicht der einzige Eignungsgrund sein. Im nachfolgenden Abschnitt wird die Eignung des Smartphones für den PU daher genauer diskutiert. In den sich anschliessenden Kapiteln wird auf Grundlage des funktionellen Umfangs eines Smartphones und fachdidaktischer Forschungsliteratur eine passende Lernumgebung herausgearbeitet. Darauf wird die Lernumgebung «FELS» (Forschend-Entdeckendes Lernen mit dem Smartphone) als Synergie der einzelnen Theoriestränge eingeführt und beispielhaft erklärt. Als weitere Legitimierung wird FELS als Unterrichtsmethode mit den Anforderungen aus den KMK-Bestimmungen (2016) verglichen.

\section{Experimente mit Smartphones im Physikunterricht}

Seit einigen Jahren werden Smartphoneexperimente im Rahmen des PUs durchgeführt und deren Nutzung durch Lehrerfortbildungen weiter forciert (u.a. Bad Honnef 2008, 1. Mitteldt. MINT Tagung 2015, SINUS Landestagung Thüringen 2015). Zumeist bleibt es bei diesen Experimenten bei einem Austausch gängiger Gerätschaften durch das Smartphone (u.a. Castro-Palacio, Velázquez-Abded, Giménez, und Monsoriu 2013; Kuhn und Vogt 2013). Zuweilen werden auch weitere Smartphonefunktionen wie Fotografieren oder Videodreh bei Experimenten genutzt (Rath und Schittelkopf 2011).

Bei dieser Art der Neugestaltung von Experimenten gilt es aber zu bedenken, dass Medien Träger von Informationen darstellen und der Lerneffekt nicht durch das Medium an sich, sondern durch die mit Hilfe des Mediums realisierte Lernumgebung generiert wird (nach Clark 1994 in: Urahne et al. 2000). Das steht mit dem Ausdruck «Kulturressource» (Bachmair, Coole und Pachler 2014, 209) für die Nutzungsmöglichkeiten mobiler Geräte nicht im Widerspruch. Spang (2014) formuliert hierzu einen 
Anspruch bezüglich der Nutzung neuer Medien. Es gehe gerade «nicht um die Abbildung herkömmlicher Methoden und Bewertungskriterien im digitalen Raum» (ebd., 112), sondern um eine zum digitalen Medium passende Lernkultur. Auf den PU bezogen muss das Experimentieren als typische Lernumgebung auf neue Medien adaptiert werden.

Experimente verfolgen dabei im schulischen PU hauptsächlich vier Zielkategorien, nämlich die Verbindung von Theorie und Praxis, den Erwerb experimenteller Fähigkeiten, das Kennenlernen wissenschaftlicher Denkweisen sowie Motivationssteigerung und persönliche Weiterentwicklung (Hucke 1999). Diese groben Zielkategorien lassen sich bei Bedarf auch feiner aufspalten (z.B. Sander 2000; Jonas-Ahrend 2004; Börlin 2012). Unter Experimentieren wird neben der Durchführung der Experimente auch deren Vor- und Nachbereitung verstanden, die wiederum ausdifferenziert sein können (Emden und Sumfleth 2012). Frischknecht-Tobler und Labudde (2010) haben für ihren Experimentierkreislauf (Abb. 1) folgende Schritte identifiziert. Als erstes stellen die Schülerinnen und Schüler Fragen an ein Phänomen oder einen Gegenstand. Aus diesen Fragestellungen heraus werden Hypothesen als Behauptung aufgestellt. Zu deren Überprüfung wird ein Experiment geplant, durchgeführt und ausgewertet. Idealerweise werden aus den Ergebnissen der Auswertung Schlussfolgerungen gezogen, um die Hypothesen zu überprüfen. Zum Schluss sollte über die Vorgehensweise beim Experimentieren und über die Ergebnisse diskutiert werden, sodass das Experiment evaluiert und gegebenenfalls erweitert werden kann.

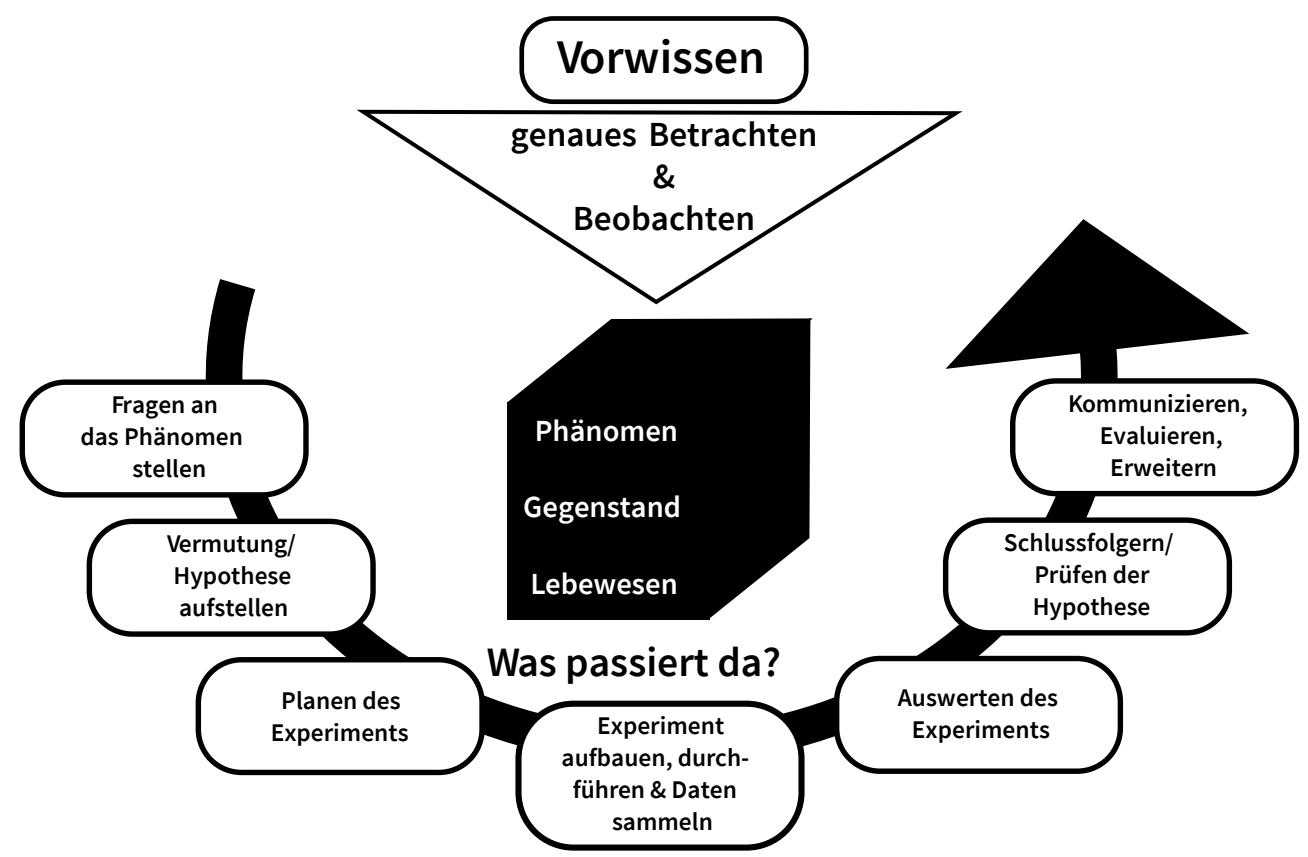

Fig. 1.: Experimentierkreislauf nach Frischknecht-Tobler und Labudde 2010, 135. 
Um Smartphones im Sinne des von Spang (2014) formulierten Anspruchs für den PU nutzbar zu machen, ist ihre Verwendung in den Experimentierkreislauf einzubetten. Diese einzelnen Schritte finden sich in ähnlicher Nomenklatur auch in den Bildungsstandards der KMK im Bereich «Erkenntnisgewinnung» (KMK 2005, 7ff) sowie in den Rahmenlehrplänen der Länder (u.a. MBJS 2015, 19ff.) wieder. Aus diesem Grund ist es wichtig, neben dem Fachwissen auch die einzelnen Teilkompetenzen der so genannten experimentellen Kompetenz (Theyßen et al. 2016) vergleichend zu testen.

Aufgrund der Vernetztheit der einzelnen Schritte im Kreislauf ist es sinnvoll, diese zu dokumentieren. Das Smartphone bietet verschiedene Möglichkeiten. Notizen und Audiokommentare können bei jedem Schritt genutzt werden. Fotos und Videos können die Durchführung dokumentieren (Kuhn, Vogt, und Müller 2011). Die am meisten genutzte Funktion des Smartphones beim Experimentieren dürfte aber die des Aufnehmens von Messwerten sein. Neben den bekannten Beschleunigungssensoren (Kuhn et al. 2011) gehören auch Gyroskop, Magnetfeldsensor, GPS, Luxmeter, Mikrofon, Kamera, Barometer sowie vereinzelt Wärmebildkameras zur Ausstattung. Mit dieser Bandbreite an Sensoren können Experimente in verschiedenen physikalischen Themenfeldern durchgeführt werden. Diese Daten können mit einfachen Notizen und Berechnungen auf dem Gerät gespeichert, online gestellt oder via email an den heimischen PC übermittelt werden.

Die handliche Grösse, die Akkutechnik und die häufig vorkommenden InternetFlat-Verträge der Jugendlichen (Jim-Studie 2015) erlauben einen mobilen und vom Fachraum unabhängigen Einsatz. Das Smartphone als mobiles Messgerät ermöglicht es, Phänomene an Gegenständen aus der möglichen Erfahrungswelt der Lernenden physikalisch zu untersuchen und letztlich zu erklären. Bei Physics 2 go (Vogt, Kasper, und Müller 2014) werden gemäss dieser Idee lebensweltliche Experimente durchgeführt. Von der Bestimmung der Schwingungsmoden einer Kirchenglocke bis zur akustischen Bestimmung von Geschwindigkeiten sind verschiedene Experimente vorgeschlagen worden. Daneben gibt es aber auch einzelne eventartige Vorschläge, innerhalb eines Klassenausflugs einen Wasserpark zu besuchen und entsprechende Messungen durchzuführen (Cabeza, Rubido, und Marti 2014) sowie beim Fahrradfahren oder Seifenkistenrennen Routen und Geschwindigkeiten über die GPS-Daten berechnen und auswerten zu lassen (Kruse 2014).

Die Kolumne IPHYSICSLABS (z.B. Kuhn, Vogt, und Theilmann 2016) hingegen stellt regelmässig Smartphoneexperimente bzw. smartphonebasierte Messmethoden vor und zeigt Lehrkräften einfach zu implementierende Beispiele auf, den eigenen Unterricht moderner zu gestalten. Klassische Experimente werden entweder moderner durchgeführt oder bislang rein theoretische Betrachtungen experimentell unterstützt. Auch altbekannte Experimentiermöglichkeiten, für die bislang ein PC nötig war, werden mit Smartphones mobil. So gibt es Videoanalyse-Apps (Becker et al. 2017) und auch per bluetooth an das Smartphone koppelbare externe Sensorpakete 
(vgl. phyphox 2018). Darüber hinaus werden für Tablet-PCs aktuell Apps beworben, die wie digitale Arbeitsbücher fungieren und multimedial das Lernen unterstützen (Huwer und Eilks 2017). Aber auch augmented reality Ansätze werden aktuell entwickelt (Thyssen 2017). Dabei werden Zusatzinformationen eingeblendet bzw. Modellvorstellungen visualisiert und naturwissenschaftliche Vorgänge begreifbarer gestaltet.

Die verschiedenen Ansätze smartphonegestützter Experimente verdeutlichen noch einmal die Variabilität und die Nützlichkeit des Smartphones für den Physikunterricht. Um jedoch das volle Potential auszuschöpfen, gilt es künftig die grundlegenden Ideen bisheriger Ansätze zu destillieren und bestmöglich miteinander zu verknüpfen.

\section{Forschend-entdeckendes Lernen}

Gemäss dem gemässigten Konstruktivismus basiert Lernen auf der Neu- und Einordnung von Wissen in das Vorwissen (de Witt und Czerwionka 2007). Tesch (2005) spezifiziert diese Aussage für den PU und schreibt, dass die Form der jeweiligen unterrichtlichen Einbettung des einzelnen Experiments bestimmte Ziele unterstützt und «für bestimmte Inhalte besonders geeignet» (Tesch 2005, 106) sei. Barth (2014) erweitert die unterrichtliche Einbettung zu übergeordneten Strategien, wodurch Experimente als «Eckpunkte in der Anlage von Unterricht zum Erreichen spezieller Zielsetzungen» (Barth 2014, 226) dienen und ermöglichen, prozessbezogene Kompetenzen in den Unterricht einzuplanen. Im Folgenden werden einige Bedingungen für eine solche von Barth (2014) geforderte übergeordnete Rahmenstruktur diskutiert.

Mit dem forschend-entdeckenden Lernen werden durch die selbstständigen Schülertätigkeiten in authentischen Situationen motivierende (Edelson et al. 1999) und durch die Prozessfokussierung (Schwab 1960) Kompetenz fördernde Aspekte verbunden. Neuere Ergebnisse (Winkelmann 2015) deuten jedoch daraufhin, dass diese positiven Effekte nicht automatisch durch offenere Unterrichtsformen entstehen, sondern weitaus abhängiger von der Lehrkraft sind. Demnach seien offenere Experimentiersettings zu überfordernd und hätten nur für leistungsstarke Schülerinnen und Schüler leicht positive Effekte (Winkelmann 2015).

Das forschend-entdeckende Lernen besitzt dennoch das Potential einer methodischen Rahmenstruktur (siehe oben), da durch die Mitbestimmung der Schülerinnen und Schülern ein natürlicheres und ungezwungeneres Aufgreifen eines Themas im Verhältnis zu deren Erfahrungswelt möglich ist als bei einem durch die Lehrkraft oktroyierten Experiment. Ausserdem ist die im normalen Unterricht oft ausgesparte Reflexionsphase unabdingbar. In der Reflexionsphase sollte auch eine Erweiterung der Erkenntnisse stattfinden, die Ergebnisse in den bisherigen Kenntnisstand einordnet und einen Ausblick auf den nachfolgenden Unterricht gibt. Dieser vernetzende 
Charakter sollte mit gestuften Hilfen auch lernschwächeren Schülerinnen und Schülern zugänglich gemacht werden. Die Lehrkraft kann dabei die Offenheit zugunsten einer besseren Strukturierung und Führung der Schülerarbeit (Banchi und Bell 2008) reduzieren.

Verschiedene historisch gewachsene Konzepte, z.B. genetischer Unterricht nach Wagenschein oder forschend-entwickelnder Unterricht nach Schmidtkunz und Lindemann, wurden immer wieder systematisierend unter dem Begriff «forschender Unterricht» zusammengeführt (u.a. Duit, Häussler, und Kircher, 1981). Reitinger (2014, 186f) leitet letztlich TILA, Theory of Inquiry Learning Arrangements, ab, benutzt aber in Anlehnung an die englische Bezeichnung inquiry based learning den Begriff «Forschendes Lernen» (Reitinger 2014). Die Begriffe Unterricht und Lernen bezeichnen nur die Sichtweisen des Lehrenden beziehungsweise der Lernenden und keine verschiedenen Modelle. Wegen der inhaltlichen Ausrichtung von TILA wird sie in Abgrenzung zu anderen Untermengen von «forschendem Unterricht» wie forschend-entwickelnder Unterricht (Schmidkunz und Lindemann, 1999) als «forschend-entdeckendes Lernen» bezeichnet.

TILA besteht aus drei Säulen. Die erste Säule beinhaltet Kriterien, die als Summe von Eigenschaften eine auf heutige Verhältnisse passende Definition von forschendentdeckendem Lernen bilden. Nach Reitinger $(2014,45)$ ist forschendes Lernen ein Prozess, in dem die «forschungsbezogenen Handlungsdomänen a) erfahrungsbasiertes Hypothetisieren, b) authentisches Explorieren, c) kritischer Diskurs und d) conclusiobasierter Transfer kennzeichnend» sind. Das Entdeckungsinteresse der Lernenden und die Methodenaffirmation stellen dabei Voraussetzungen dar (ebd.). Unter Methodenaffirmation wird verstanden, dass der Forschungsprozess als echt empfunden werden muss, d.h. er ist durch den Lernenden gewollt und zumindest teilweise mitbestimmt. Das authentische Explorieren meint ein selbstgesteuertes Experimentieren in für den Lernenden bedeutungsvollen Kontexten. Beim kritischen Diskurs werden die Ergebnisse, der Erkenntnisprozess sowie die dabei entstandenen Bedeutungszuweisungen reflektiert. Der conclusiobasierte Transfer ist die logische Folgerung, bei gleicher Ausgangslage und gleichem Ergebnis das dahinterliegende gemeinsame Prinzip übertragen zu können. Dieser kann in zwei Formen geschehen, nämlich in einem strukturellen Transfer in andere Kontexte oder in einer Veröffentlichung der durchgeführten Untersuchung in Form eines Vortrags oder eines Posters.

Die zweite Säule von TILA beinhaltet handlungsleitende Vorschläge in Form von unterrichts- und lernprozessfördernden Prinzipien: Vertrauen, Selbstbestimmung, Veranschaulichung, Sicherheit, Strukturierung sowie Personalisierung. Sie müssen durch den Vorschlagscharakter innerhalb einer Lernumgebung nicht allumfassend erfüllt sein (Reitinger 2014). Schülerinnen und Schüler, die offene Lernarrangements nur selten bis nie erlebten, werden ohne Hilfe der Lehrkraft bei der Bearbeitung Probleme bekommen oder gar scheitern. Aus diesem Grund sind Sicherheit und 
Strukturierung zwei der sieben Prinzipien. Die Struktur gibt ein Grundgerüst vor, an dem sich die Lernenden orientieren können und reduziert die Gefahr des Verrennens in offenen Lerngelegenheiten (vgl. Wahser und Sumfleth 2008). Dabei soll nicht das selbstgesteuerte Vorgehen strukturiert und zerstört werden, sondern die Lernenden werden innerhalb der Prozessstruktur durch ein Lernarrangement geleitet, das durch die bereits genannten Kriterien und Prinzipien Orientierung bietet (Reitinger 2014). Sicherheit ist in dieser Hinsicht enorm wichtig und wird durch Materialsammlungen, Hilfen oder auch der Anwesenheit der Lehrkraft erzeugt und stellt letztlich Scaffolding dar. Scaffolding ist dabei keine Neuentwicklung, sondern ein bewährtes Mittel, Schülerinnen und Schüler in offenen Lernformen zu unterstützen und Überforderungen zu vermeiden (vgl. Hmelo-Silver et al. 2007). In Anlehnung an die gestuften Hilfen bei Lernaufgaben von Leisen (1999), Franke-Braun et al. (2008) und Wodzinski (2013) kann von einer Form der Binnendifferenzierung und der Förderung des selbstständigen, kooperativen Lernens sowie fachbezogener Selbstwahrnehmungen ausgegangen werden. Trotz des teilweise offenheitseinschränkenden Charakters des Scaffolding erfüllt es Kriterien autonomiefördernden Unterrichts (Hofferber et al. 2015).

Die dritte Säule von TILA berücksichtigt die Lehrerseite in Form des systemischen Organisationsmodells OPeRA (Reitinger 2012), nachdem der Unterricht entworfen, durchgeführt und reflektiert wird.

\section{Kontexte}

Für einen gemäss den Bildungsstandards (KMK 2005) kompetenzorientierten PU sind neben Methoden auch Inhalte wichtig, weil Kompetenzen in konkreten Situationen an konkreten Inhalten erlernt werden. Entsprechend wichtig ist der Kontext, in dem das Lernen thematisch stattfindet (Schacter 1996). Beim forschend-entdeckenden Lernen gibt es in zwei verschiedene Kontexte, einen sozialen Kontext mit Lernenden als kleine Forschern, sowie einen inhaltlichen Kontext (Höttecke und Rieß 2015). Van Vorst et al. (2014) haben sich bewusst nur auf den letzten Aspekt konzentriert, indem sie Kontext als «Verflechtung fachlicher Inhalte und Zugänge [definieren; Anm. d. A.], die aus nichtfachlicher Sicht betrachtet werden» (Van Vorst et al. 2014, 30). Durch diese Verflechtungen finden Bedeutungszuweisungen statt und es können Emotionen geweckt werden. Besondere Berücksichtigung bei der Kontextwahl muss daher neben der Authentizität die Bekanntheit haben (Van Vorst et al. 2014). Der Kontext Staumauer wäre demnach zum Erlernen der physikalischen Grösse Druck nur dann sinnvoll, wenn die Lernenden diese aus ihrer eigenen Lebenswelt kennen. In Brandenburg, wo es solche Staumauern kaum gibt, bieten die weitaus häufigeren Schleusen den besseren Anknüpfungspunkt. Sollten die Schülerinnen und Schüler als Kinder beim Paddeln staunend durch Schleusen gefahren sein, ruft dieser Kontext bei diesen Schülerinnen und Schülern positive Emotionen wach und weckt idealerweise 
auch Neugier, wie eine Schleuse funktioniert. Darüber hinaus wirken sich Emotionen wie Freude und Interesse in Orientierungs- und Lernphasen positiv auf Lernleistung aus, sofern es frei von äusseren Zwängen oder Druck abläuft (Laukenmann 2000).

Bisherige Ansätze wie PIKO (Mikelskis-Seifert und Duit 2010) oder die Arbeit mit Ankermedien wie Zeitungsartikel (Kuhn 2010) gehen insbesondere bei der Authentizität noch nicht weit genug. Lebensweltbezug im Sinne eines Kontextes bedeutet die inhaltliche Verschiebung von einer reinen Fachsystematik im Fachraum hin zu einer Anknüpfung an ausserunterrichtliche Erfahrungen der Lernenden. Oftmals werden aber dabei Problemfelder konstruiert, die für die Lernenden keine darstellen, indem z.B. nach physikalischen Ursachen für Supermans übermenschlicher Kraft gefragt wird (Kuhn 2011). Jedoch scheinen einige Kontexte besonders positiv zu sein (IPN Interessensstudie), während sich andere von biographischen Elemente der Lernenden ableiten lassen. Am Ende obliegt es der jeweiligen Lehrkraft, geeignete Kontexte auf Basis der Kenntnis der einzelnen Klasse auszuwählen.

Der Kritikpunkt der «synthetischen Wirklichkeit» (Hagelgans 2015, 2) am PU bleibt. Gemäss dem situierten Lernen findet das Lernen in der jeweiligen Situation, einschliesslich der sozialen Umgebung und dem Lernort statt (de Witt und Czerwionka 2007). Dadurch stellt der Lernort selbst einen Teil des Kontexts dar. Zusammen mit der Feststellung von Bernhard (2015, 5): «Wir sehen nur das, was wir mit Begriffen benennen können. Umgekehrt kommen wir gedanklich nicht weiter, wenn wir von Begriffen keine Anschauung haben» führt dies zur Forderung nach ausserschulischen Lernorten, wo die Lernenden die Gegenstände aus dem eigenen Alltag nicht nur kennen, sondern diese auch anfassen und für Experimente passend manipulieren können. Dabei hilft auch eine natürliche Umgebung, die verschiedene Sinne ansprechen kann und Objekte in ihrem Wirkzusammenhang untersuchen lässt (Smeds et al. 2015). Smeds et al. (2015) fanden heraus, dass der Lernstand in dem ausserschulischen Lernort im follow-up-Test signifikant besser war als in den Kontrollgruppen, die nicht oder nur teilweise den ausserschulischen Lernort besuchten, wobei die Lerngelegenheiten am ausserschulischen Lernort als persönlich bedeutsam eingeschätzt wurde. Der ausserschulische Lernort kam dabei nicht nur guten, sondern auch weniger guten Schülern zugute. Auch in der Chemie werden die Vorteile ausserschulischer Lernorte genutzt. Engl und Risch (2015) verlagern bewusst Experimente in die Natur, um den scheinbaren Widerspruch zwischen Natur und Chemie aufzulösen. Übertragen auf die Physik kann es sich also nur um ausserschulische, authentische Experimente handeln, die von den Lernenden ausgeführt werden. Bei dem Stundenkontingent von PU ist dies nicht mit Ausflügen, sondern durch kleine Hausaufgaben realisierbar. 


\section{Forschend-Entdeckendes Lernen mit dem Smartphone (FELS)}

FELS bietet eine Lernkultur, die für Jugendliche eine Verbindung zwischen ihrem Alltag und dem PU schaffen kann. Sie lässt die eigene Lebenswelt durch eine physikalische Brille mit Hilfe von Smartphoneexperimenten scheinen. FELS ist in Anlehnung an den Experimentierkreislauf (Frischknecht-Tobler und Labudde 2010) ein 6-phasiger Lernzyklus (Abb. 2) und entspricht einer stressfreien Erarbeitungsphase (Laukenmann et al. 2000), wobei durch die Nutzung eines blended-learning Konzepts trotz der Hausaufgaben eine unterbrechungsfreie, gesteuerte Lernsituation initiiert wird (Specht et al. 2013). Das blended-learning beginnt mit einer für die Lernenden authentischen Initialisierung innerhalb der Klasse. Mit mobilen Geräten werden dann persönliche Lernerfahrungen ausserhalb des Unterrichts gemacht, wobei die Schülerinnen und Schüler einerseits aktiv beobachten und andererseits kritisch reflektieren. Über Online-Plattformen tauschen sie sich aus und bewerten einander, bevor im Klassenverband eine Wissenssicherung im Klassenraum vorgenommen wird.

FELS - Forschend-Entdeckendes Lernen mit dem Smartphone

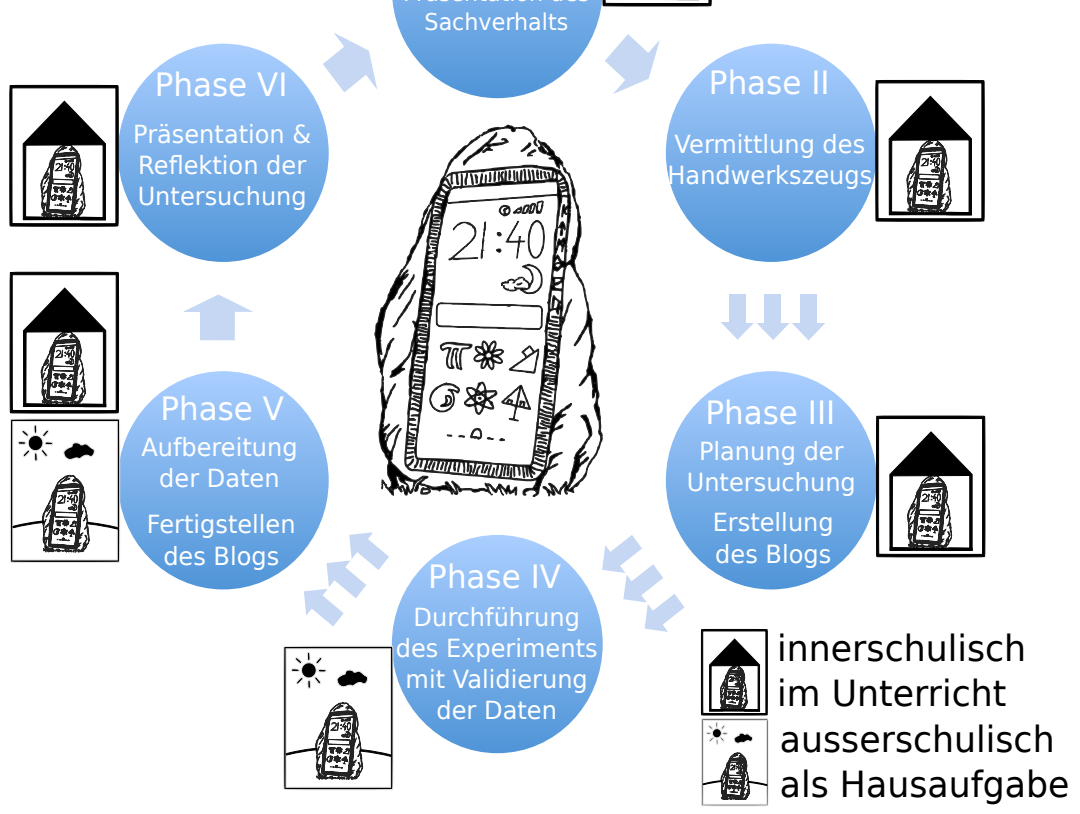

Fig. 2.: Der 6-phasige Lernzyklus FELS nach Müller, Magdans und Borowski 2016, 231.

Im Folgenden ist ein möglicher Unterrichtsverlauf zum Federschwinger unter Angabe der Lernziele tabellarisch skizziert. Dabei sind der Verlauf sowie der Medieneinsatz prototypisch, die Lernziele hingegen stellen eine beispielhafte Auswahl dar. 


\begin{tabular}{|c|c|c|c|c|c|}
\hline Verlauf & $\begin{array}{l}\text { Phase } \\
\text { von } \\
\text { FELS }\end{array}$ & Lehrertätigkeit & Schülertätigkeit & Medieneinsatz & Zielsetzung \\
\hline \multirow[t]{2}{*}{ Stunde 1} & 1 & $\begin{array}{l}\text { - Macht Sequenz trans- } \\
\text { parent } \\
\text { - Zeigt Video eines } \\
\text { Mannes, der auf einem } \\
\text { Freischwinger wippt } \\
\text { - Leitet Brainstorming } \\
\text { an, sammelt Antwor- } \\
\text { ten, clustert diese und } \\
\text { ergänzt ggf. }\end{array}$ & $\begin{array}{l}\text { - Beobachten und hö- } \\
\text { ren zu } \\
\text { - Sammeln Fragen zu } \\
\text { ihren Beobachtungen } \\
\text { des Videos }\end{array}$ & $\begin{array}{l}\text { - Sprache beim einfüh- } \\
\text { renden Lehrervortrag, } \\
\text { der Sammlung sowie } \\
\text { der Moderation } \\
\text { - Video zur Präsentation } \\
\text { des Phänomens } \\
\text { - Tafel zur Fixierung der } \\
\text { einzelnen Fragestellun- } \\
\text { gen (Smartboard) }\end{array}$ & $\begin{array}{l}\text { - Schülerinnen und } \\
\text { Schüler zur Mitarbeit } \\
\text { innerhalb der Sequenz } \\
\text { motivieren } \\
\text { - Schülerinnen und } \\
\text { Schüler können ein } \\
\text { Phänomen beobach- } \\
\text { ten und physikalische } \\
\text { Fragestellungen unter } \\
\text { Verwendung von All- } \\
\text { tagssprache entwickeln }\end{array}$ \\
\hline & II & $\begin{array}{l}\text { - Vermittelt bzw. wieder- } \\
\text { holt Definitionen von } \\
\text { Periodendauer, Ampli- } \\
\text { tude, Beschleunigung } \\
\text { - Stellt passende Apps } \\
\text { vor (Vidanalysis free } \\
\text { und Phyphox) }\end{array}$ & $\begin{array}{l}\text { - Hören zu und arbeiten } \\
\text { mit } \\
\text { - Laden sich Apps runter } \\
\text { - } \text { Recherchieren ggf. } \\
\text { selbst weiter im In- } \\
\text { ternet }\end{array}$ & $\begin{array}{l}\text { - Sprache beim Lehrer- } \\
\text { vortrag } \\
\text { - Systematisierungen } \\
\text { und Nachschlagewerke } \\
\text { (Tafelbild der Lehrkraft, } \\
\text { Lehrbuch, Smartphone } \\
\text { mit Internet) } \\
\text { - Notizen (im Hefter, im } \\
\text { Smartphone) }\end{array}$ & $\begin{array}{l}\text { Schülerinnen und } \\
\text { Schüler können einen } \\
\text { Federschwinger mit } \\
\text { Hilfe ihres Smartpho- } \\
\text { nes vermessen }\end{array}$ \\
\hline Stunde 2 & III & $\begin{array}{l}\text { - Teilt Klasse in } 8 \text { Grup- } \\
\text { pen ein } \\
\text { - Teilt Aufgaben aus, je } 2 \\
\text { Gruppen erhalten die } \\
\text { gleiche Aufgabe } \\
\text { - Geht herum, hilft ggf. } \\
\text { bei der Aufgabenbear- } \\
\text { beitung }\end{array}$ & 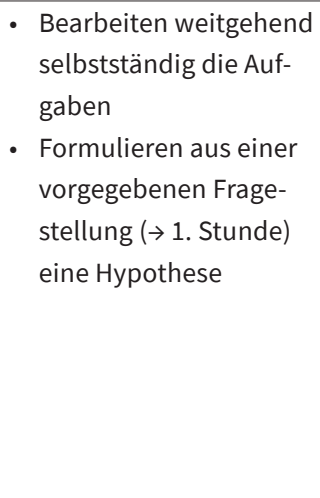 & $\begin{array}{l}\text { - Sprache bei der Erar- } \\
\text { beitung des Experi- } \\
\text { mentierplans innerhalb } \\
\text { der einzelnen Gruppen } \\
\text { - Aufgabenblatt oder } \\
\text { FELS-App auf dem } \\
\text { Smartphone mit Einga- } \\
\text { beaufforderungen }\end{array}$ & $\begin{array}{l}\text { - Schülerinnen und } \\
\text { Schüler können an- } \\
\text { hand eines Beispiels } \\
\text { aus einer Fragestellung } \\
\text { eine Hypothese in } \\
\text { Form eines physika- } \\
\text { lischen Zusammen- } \\
\text { hangs zweier Grössen } \\
\text { aufstellen und einen } \\
\text { Plan zur experimen- } \\
\text { tellen Untersuchung } \\
\text { aufstellen. }\end{array}$ \\
\hline
\end{tabular}




\begin{tabular}{|c|c|c|c|c|c|}
\hline Verlauf & $\begin{array}{l}\text { Phase } \\
\text { von } \\
\text { FELS }\end{array}$ & Lehrertätigkeit & Schülertätigkeit & Medieneinsatz & Zielsetzung \\
\hline \multirow[t]{2}{*}{$\begin{array}{l}\text { Hausauf- } \\
\text { gabe }\end{array}$} & IV & I & $\begin{array}{l}\text { - Führen geplantes } \\
\text { Experiment durch, } \\
\text { z.B. Wippen auf einem } \\
\text { Sitzball zur Prüfung } \\
\text { der Hypothese «Die } \\
\text { Periodendauer nimmt } \\
\text { zusammen mit der } \\
\text { Amplitude ab» }\end{array}$ & $\begin{array}{l}\text { - Sprache zur Koordinie- } \\
\text { rung der Aufgabenbe- } \\
\text { wältigung innerhalb } \\
\text { der Gruppen } \\
\text { - Messapp auf dem } \\
\text { Smartphone, z.B. Phy- } \\
\text { phox zur Messung der } \\
\text { physikalischen Grössen } \\
\text { Zeit und Beschleuni- } \\
\text { gung (hier: als Mass für } \\
\text { die Amplitude) Zeit } \\
\text { - Untersuchungsblog als } \\
\text { einsehbare Mischform } \\
\text { aus Lerntagebuch und } \\
\text { Protokoll }\end{array}$ & $\begin{array}{l}\text { - Schülerinnen und } \\
\text { Schüler können selbst- } \\
\text { ständig ein Experiment } \\
\text { durchführen und doku- } \\
\text { mentieren }\end{array}$ \\
\hline & V & $\begin{array}{l}\text { - Liest Untersuchungs- } \\
\text { blogs } \\
\text { - Gibt Hilfestellung via } \\
\text { Kommentar-funktion }\end{array}$ & $\begin{array}{l}\text { - Werten gesammelte } \\
\text { Daten aus } \\
\text { - Bestätigen bzw. (hier) } \\
\text { widerlegen Hypothese } \\
\text { - Vervollständigen Un- } \\
\text { tersuchungsblog } \\
\text { - Kommentieren die } \\
\text { Untersuchungsblogs } \\
\text { der anderen }\end{array}$ & $\begin{array}{l}\text { - Tabellenkalkulations- } \\
\text { programm am PC zur } \\
\text { feineren Auswertung } \\
\text { der auf der App ange- } \\
\text { zeigten Diagramme } \\
\text { - Untersuchungsblog } \\
\text { am PC als einsehbare } \\
\text { Mischform aus Lernta- } \\
\text { gebuch und Protokoll } \\
\text { sowie Kommentierung } \\
\text { anderer als Hilfestel- } \\
\text { lung }\end{array}$ & $\begin{array}{l}\text { - Schülerinnen und } \\
\text { Schüler können ein } \\
\text { selbst durchgeführtes } \\
\text { Experiment auswerten } \\
\text { und ihr Vorgehen doku- } \\
\text { mentieren } \\
\text { - Schülerinnen und } \\
\text { Schüler sind in der } \\
\text { Lage, Kritik an ihrem } \\
\text { Vorgehen einzuarbei- } \\
\text { ten. }\end{array}$ \\
\hline Stunde 3 & $\mathrm{VI}$ & $\begin{array}{l}\text { - Moderiert Präsenta- } \\
\text { tionen der einzelnen } \\
\text { Gruppen, indem er die } \\
\text { Diskussionen leitet und } \\
\text { die Untersuchungs- } \\
\text { blogs der einzelnen } \\
\text { Gruppen evaluiert so- } \\
\text { wie zu einem Gesamt- } \\
\text { bild zusammenfasst } \\
\text { - Leitet zum nächsten } \\
\text { Thema ausblicksartig } \\
\text { über }\end{array}$ & $\begin{array}{l}\text { - Präsentieren ihre je- } \\
\text { weilige Gruppenarbeit, } \\
\text { z.B. die Untersuchung } \\
\text { eines Federschwingers } \\
\text { am Beispiel eines } \\
\text { Sitzballs } \\
\text { - Diskutieren den Er- } \\
\text { kenntnisprozess sowie } \\
\text { die Ergebnisse der } \\
\text { anderen Gruppen } \\
\text { - Geben Ratschläge für } \\
\text { Verbesserungen } \\
\text { - Hören zu und überneh- } \\
\text { men das Tafelbild }\end{array}$ & $\begin{array}{l}\text { - Untersuchungsblog als } \\
\text { Präsentationsgrundla- } \\
\text { ge mit PC und Beamer } \\
\text { - Tafel (Smartboard) zur } \\
\text { Zusammenfassung } \\
\text { aller Beiträge } \\
\text { - Hefter / Foto mit dem } \\
\text { Smartphone zur Siche- } \\
\text { rung des Tafelbildes } \\
\text { - Sprache bei der Prä- } \\
\text { sentation, Diskussion } \\
\text { und dem Lehrervortrag }\end{array}$ & $\begin{array}{l}\text { Schülerinnen und } \\
\text { Schüler können eine } \\
\text { selbst geplante und } \\
\text { durchgeführte Unter- } \\
\text { suchung präsentieren } \\
\text { und ihre Ergebnisse } \\
\text { sowie Vorgehenswei- } \\
\text { sen unter Verwendung } \\
\text { von Fachsprache dis- } \\
\text { kutieren }\end{array}$ \\
\hline
\end{tabular}


In der ersten Phase, die im regulären PU innerhalb des Klassenraumes stattfindet, steht ein physikalischer Sachverhalt als «Phänomen» im Mittelpunkt. Der physikalische Sachverhalt in Abgrenzung zum Phänomen bedeutet, dass der physikalische Prozess gut erkennbar sein sollte, um diesen in Alltagsexperimenten wiederfinden zu können, zu erweitern oder zu präzisieren. Höttecke und Rieß (2015) bezeichnen dies als «Erscheinung» (Höttecke und Rieß 2015, 130). Mit der Präsentation des physikalischen Sachverhalts in Form eines Demonstrationsexperiments sollen die Schülerinnen und Schüler motiviert werden, innerhalb des Brainstormings Fragestellungen zu entwickeln, was dort passiere. Diese werden an der Tafel fixiert und wenn nötig von der Lehrkraft ergänzt. Anzumerken ist, dass Phänomen-Erklärungen mit Hilfe von Experimenten mit 50\% den dritten Platz der beliebtesten Unterrichtsinhalte in Physik darstellen (Merzyn 2015). Zusätzlich möchten die Schülerinnen und Schüler die Sinnhaftigkeit des Unterrichts erkennen und fordern Alltagsgeschehen und Phänomene ein, was bei FELS an späterer Stelle folgen wird. Das Aufstellen der Fragestellungen entspricht einer Eigenaktivität der Lernenden, was zu Interessenssteigerungen führen kann (Merzyn 2015). Die Fixierung der durch die Lernenden entwickelten Fragestellungen verfolgt drei Ziele. Die Schülerinnen und Schüler bekommen erstens das Gefühl, selbst einen Teil des Unterrichts mitzugestalten und dabei ernst genommen zu werden. Ausserdem gibt es hier für Lehrkräfte die Möglichkeit, Gruppen zur Bearbeitung zu organisieren und zu planen (Streller 2013). Drittens erhält die Lehrkraft die Gelegenheit, mögliche den Lernprozess behindernde Präkonzepte zu diagnostizieren, um in späteren Phasen darauf eingehen zu können.

Phase II stellt einen Einschub dar. Die Lernenden benötigen ein Minimum an theoretischem Input. Die Experimente sind hypothesengeleitet (Frischknecht-Tobler und Labudde 2010) und die Schülerinnen und Schüler sollten wissen, was genau sie wie mit dem Smartphone messen oder aufzeichnen können. Ausserdem benötigen die vier schriftlichen, aufgabenbasierten Scaffoldings, die auf den Fragestellungen aus Phase I basieren und mit deren Hilfe in Phase III ein Experimentierplan entstehen soll, eine gewisse Vorbereitungszeit der Lehrkraft.

Phase III ist nicht als Frontalunterricht, sondern als Gruppenarbeit ausgelegt. Dabei wird evolutionär vorgegangen (Widodo und Duit 2005). Die Lernenden beginnen bei ihrer zum Teil in Alltagssprache formulierten Fragestellung aus Phase I und werden über aufgabengeleitetes Scaffolding zur Hypothesenbildung und schliesslich zu einem Experimentierplan angeleitet. Durch die schriftlichen Scaffoldings bleibt der Lehrkraft genug Zeit, um bei Fragen oder Problemen den einzelnen Gruppen zu helfen. Ein rein mündliches Scaffolding oder ein Klassengespräch scheiden aus, da verschiedene Teilexperimente zu einem Sachverhalt von den Schülerinnen und Schülern erarbeitet werden sollen und die Lehrkraft nicht mit allen gleichzeitig reden kann. Um jeden Lernenden bzw. jede Gruppe ernst zu nehmen, werden in einer durchschnittlichen Klasse vier verschiedene Fragestellungen benötigt. Zwei Gruppen 
bearbeiten jeweils die gleiche Fragestellung, können aber durchaus zu leicht anderen Hypothesen beziehungsweise Experimentierplanungen gelangen. Dadurch wird ein Thema von vier Facetten aus beleuchtet und es gibt jeweils eine Gruppe, die der anderen hilfreiche Tipps in den Phasen IV und V geben kann. Auch ist die Lehrkraft im Stande, den Erkenntnisprozess in Phase VI zu beleuchten, weil jeweils zwei leicht verschiedene Experimente zur gleichen Fragestellung erarbeitet werden. Bei den Planungen ist darauf zu achten, dass die einzelnen Experimente innerhalb von 15 Minuten durchführbar sind. Wegen der inhaltlichen Verantwortung durch die Schülerinnen und Schüler muss die Organisation in diesem Fall durch die Lehrkraft erfolgen (Sumfleth und Nicolai 2009).

Die geplanten, ausserschulischen Smartphoneexperimente führen die Schülerinnen und Schüler innerhalb ihrer Gruppen in Phase IV als Hausaufgaben durch. An dieser Stelle sei darauf hingewiesen, dass Hausaufgaben nicht unumstritten sind (u.a. Kohler 2011; Sumfleth et al. 2011). In der Schulpraxis haben sie häufig den Charakter einer Strafarbeit (Schwemmer 1980). Lernende, die wegen eines schlechten Zeitmanagments oder durch ein kollektives Klassenverhalten den entsprechenden Inhalt nicht innerhalb der Unterrichtszeit bearbeiten konnten, müssen diesen zu Hause nachholen. Auch spiegeln Hausaufgaben weniger den Leistungsstand des Lernenden, sondern mehr die Korrekturfähigkeit der Eltern sowie der Nachhilfe wieder und erhöhen die soziale Ungerechtigkeit von Schule (Nieswand 2014). Die Lernenden bearbeiten aber nicht normale Hausaufgaben, sondern sie führen den Unterricht an einem anderen Ort durch. West und Vosloo $(2013,18)$ merken hierzu an: «Mobile devices can [...] give literal meaning to the maxim 'the world is a classroom '.» Da diese experimentellen Aufgaben so in der Schule nicht möglich wären, handelt es sich auch um keine Strafarbeit, sondern um eine bewusste didaktische Entscheidung. Durch diesen Einschub des mobile-learning können sich die Schülerinnen und Schüler ihre Bearbeitungszeit frei einplanen, was den Druck mindert (de Witt 2013; West und Vosloo 2013). Dabei werden Lernkontexte mit Hilfe des Smartphones erzeugt, in denen die Schülerinnen und Schüler selbstständig Wissen generieren, wobei das Smartphone nicht nur als Experimentiermittel, sondern auch als Werkzeug zur Kommunikation und Individualisierung dient (Bachamar, Coole, und Pachler 2014). Ausserdem ist davon auszugehen, dass viele der ausserschulischen Smartphoneexperimente nicht in den heimischen Wänden erledigt werden können und somit kein Bewegungsmangel induziert wird.

Die Auswertung in Phase V kann sich dabei entweder an das Experimentieren zu Hause in Phase IV anschliessen, oder findet je nach Bedürfnissen der Lehrkraft im Unterricht statt. Für die Schülerinnen und Schüler ist die Heimarbeit wegen der freien Zeiteinteilung bei der Erstellung der Präsentation für Phase VI als positiv zu benennen. Auf der anderen Seite kann die Lehrkraft innerhalb des Unterrichts stärker regulativ und helfend eingreifen und reduziert so den Umfang der anfallenden 
Hausaufgaben. Auch kann hierbei sicher gestellt werden, dass alle Auswertungen zeitgleich fertig sind, sodass die Schülerinnen und Schüler zu Hause die Untersuchungsblogs der anderen kommentieren können und auch noch Zeit bis zur Folgestunde wäre, die Kommentare gegebenenfalls noch in die Präsentation für Phase VI einzuarbeiten.

Alle Planungen, Beobachtungen und Ergebnisse werden in dem «Untersuchungsblog» hochgeladen, sodass die Mitlernenden und auch die Lehrkraft jederzeit die Möglichkeit haben, Kommentare und Hilfestellungen zu den einzelnen Experimenten der jeweiligen Gruppe zu geben (Grell und Rau 2011). Dies umfasst nicht nur die Ergebnisse, sondern explizit auch den Entstehungsprozess. Über eine Lernplattform wie moodle, wo alle Untersuchungsblogs entsprechend verlinkt sind, lässt sich ein virtuelles Klassenzimmer generieren. Durch diesen gegenseitigen Austausch innerhalb der einzelnen Untersuchungsblogs, kommen die Schülerinnen und Schüler in eine «Zone der proximalen Entwicklung» (nach Wygotski, 1974 zitiert nach Urahne et al. 2000, 168). Zusammen mit der Verflechtung mit dem Unterricht in Phase I, III und $\mathrm{VI}$, also die Nutzung eines blended-learning Konzepts, stellt die Nutzung der Lernplattform auch keinen didaktischen Rückschritt dar (nach Reinmann 2005 in Zumbach et al. 2014). Um die Schülerinnen und Schüler zu entlasten, sie ernst zu nehmen und um Hausaufgabenzeit zu minimieren, dient der Untersuchungsblog als Präsentationsgrundlage, weswegen die jeweilige Lehrkraft eine Gliederungsstruktur für den Untersuchungsblog vorgeben muss.

Phase VI findet im Klassenverband innerhalb des regulären Klassen- oder Physikraumes statt. Die Schülerinnen und Schüler präsentieren anhand ihres Untersuchungsblogs, was sie warum wie gemacht haben und zu welchen Daten, Ergebnissen und Schlussfolgerungen sie bezogen auf die Hypothese kamen. Darüber hinaus sollte jeweils noch kurz diskutiert und Rückfragen der Mitschüler geklärt werden. Dieses gegenseitige Erklären fördert auch den Lernzuwachs der Schülerinnen und Schüler (Rabe und Mikelskis 2007). Da immer zwei Gruppen die gleiche Fragestellung bearbeiten und dadurch ein zumindest ähnliches Experiment erarbeitet haben, gibt es auch die Möglichkeit des Vergleichs, was eine kritische Reflexion des Vorgehens erleichtern kann.

Die Phasen I, II, IV, V und VI sind dabei auch vergleichbar mit dem konstruktivistischen Referenzmodell für Lehr-Lern-Sequenzen, bei dem zunächst Schülervorstellungen erkundet werden (Widodo und Duit 2005). Bei FELS werden in Phase I Fragestellungen durch die Lernenden aufgestellt, die auf ihren Vorstellungen beruhen. Die Umstrukturierung der Schülervorstellungen findet bei FELS durch den Theorieeinschub in Phase II und der Ausarbeitung des Experiments in Phase III statt. Die Anwendung der neuen Vorstellungen entspräche dann dem Experimentieren und dem Auswerten in Phase IV und V. Das Überprüfen und Bewerten der neuen Vorstellungen findet bei FELS in Phase VI mit der Präsentation und der zugehörigen Diskussion 
statt. Allen Phasen den gleichen Stellenwert zu geben, könnte die Schülerinnen und Schüler jedoch überfordern.

Je nach Schwerpunktsetzung lässt sich auch die Produktorientierung der Schülerinnen und Schüler nutzen. Die Produktorientierung verleitet die Lernenden bei gewöhnlichen Experimenten zügig Messwerte für das Protokoll für die Lehrkraft zu produzieren, wobei alles andere untergeordnet wird (Sander 2000). Bei FELS läge der Fokus eines zu erarbeitenden Produktes aber nicht auf Messwertgenerierung, sondern auf dem im Untersuchungsblog nachvollziehbaren Experimentierplan sowie auf der Präsentation am Ende von FELS. In Anlehnung an Müller, Scharner und Henneberg (2014) könnte das vermeintliche Manko einer Produktorientierung auch bei FELS positiv genutzt werden.

\section{Abgleich mit den Bildungsempfehlungen zur Medienkompetenz}

Die KMK hat über alle Fächer querliegende Medienkompetenzen verabschiedet. Inwiefern diese ausreichen oder konkretisiert werden müssen, soll an dieser Stelle nicht diskutiert werden. Dafür sei auf entsprechende Positionspapiere wie z.B. von KBoM! bzw. der GMK (2018) verwiesen. Stattdessen soll eine exemplarische Verortung der sechs Medienkompetenzbereiche (KMK 2016) für den PU in der Lernumgebung FELS erfolgen. Aus Gründen der Übersichtlichkeit wird auf eine Darstellung der nicht mit FELS vermittelbaren Teilkompetenzen verzichtet. Eine vollständige Darstellung kann beim Autor erfragt werden.

Kompetenzbereich: Suchen, Verarbeiten und Aufbewahren

\begin{tabular}{|c|c|}
\hline Teilkompetenz & Implementierung in FELS \\
\hline $\begin{array}{l}\text { 1.2.1 «Informationen und Daten analysieren, } \\
\text { interpretieren und kritisch bewerten» }\end{array}$ & $\begin{aligned} \rightarrow & \text { Phase IV - V: Datenanalyse und Interpreta- } \\
& \text { tion bezüglich des Experiments } \\
\rightarrow & \text { Phase VI: Bewertung der Messergebnisse in } \\
& \text { Diskussion; Erleichterung der Interpretati- } \\
& \text { on durch Kenntnisse der Sensortechnik } \\
\rightarrow & \text { ABER: keine traditionelle Quellenkritik }\end{aligned}$ \\
\hline $\begin{array}{l}\text { 1.3.1 «Informationen und Daten sicher spei- } \\
\text { chern, wiederfinden und von verschie- } \\
\text { denen Orten abrufen» }\end{array}$ & $\begin{array}{l}\rightarrow \text { Phase IV - VI: Verwendung verschiedener } \\
\text { Hardware (Smartphone, PC, Smartboard) } \\
\text { während Durchführung, Auswertung und } \\
\text { Präsentation }\end{array}$ \\
\hline $\begin{array}{l}\text { 1.3.2 «Informationen und Daten zusammen- } \\
\text { fassen, organisieren und strukturiert } \\
\text { aufbewahren» }\end{array}$ & $\begin{aligned} \rightarrow & \text { Phase V: Auswertung der Daten und Erstel- } \\
& \text { lung des Untersuchungsblogs }\end{aligned}$ \\
\hline
\end{tabular}


Kompetenzbereich: Kommunizieren und Kooperieren

\begin{tabular}{|c|c|c|}
\hline \multicolumn{2}{|c|}{ Teilkompetenz } & \multirow{2}{*}{$\begin{array}{l}\text { Implementierung in FELS } \\
\rightarrow \text { Phase V: Kommentierungen der Untersu- } \\
\text { chungsblogs }\end{array}$} \\
\hline 2.1.1 & $\begin{array}{l}\text { «Mit Hilfe verschiedener digitaler Kom- } \\
\text { munikationsmöglichkeiten kommuni- } \\
\text { zieren» }\end{array}$ & \\
\hline 2.2 .1 & $\begin{array}{l}\text { «Dateien, Informationen und Links } \\
\text { teilen» }\end{array}$ & $\begin{array}{l}\rightarrow \text { Phase V: Teilen der Messergebnisse zur } \\
\text { gemeinsamen Auswertung in der Gruppe } \\
\text { sowie Veröffentlichung der Messergebnisse } \\
\text { und deren Interpretation im Untersu- } \\
\text { chungsblog }\end{array}$ \\
\hline 2.3.1 & $\begin{array}{l}\text { «Digitale Werkzeuge für die Zusam- } \\
\text { menarbeit bei der Zusammenführung } \\
\text { von Informationen, Daten und Ressour- } \\
\text { cen nutzen» }\end{array}$ & $\begin{array}{l}\rightarrow \text { Phase V: Nutzung von emails zum Daten- } \\
\text { austausch innerhalb der Gruppe, Tabel- } \\
\text { lenkalkulationen zur Datenauswertung } \\
\text { mehrerer Messreihen und Erstellung des } \\
\text { Untersuchungsblogs }\end{array}$ \\
\hline 2.4 .1 & $\begin{array}{l}\text { «Verhaltensregeln bei digitaler Inter- } \\
\text { aktion und Kooperation kennen und } \\
\text { anwenden» }\end{array}$ & $\begin{array}{l}\rightarrow \text { Phase V: Kommunikationsregeln bei der } \\
\text { Kommentierung anderer Untersuchungs- } \\
\text { blogs }\end{array}$ \\
\hline
\end{tabular}

Kompetenzbereich: Produzieren und Präsentieren

\begin{tabular}{|c|c|}
\hline Teilkompetenz & Implementierung in FELS \\
\hline $\begin{array}{l}\text { 3.2.1 «Inhalte in verschiedenen Formaten } \\
\text { bearbeiten, zusammenführen und } \\
\text { präsentieren» }\end{array}$ & $\begin{aligned} \rightarrow & \text { Phase V: Zusammenführung und Auswer- } \\
& \text { tung digitaler Messdaten in Tabellenkalku- } \\
& \text { lation sowie Protokollierung im Untersu- } \\
& \text { chungsblog } \\
\rightarrow & \text { Phase VI: Präsentation des Untersuchungs- } \\
& \text { blogs }\end{aligned}$ \\
\hline $\begin{array}{l}\text { 3.2.2 «Informationen, Inhalte und vorhande- } \\
\text { ne digitale Produkte weiterverarbeiten } \\
\text { und in bestehendes Wissen integrie- } \\
\text { ren» }\end{array}$ & $\begin{array}{l}\rightarrow \text { Phase VI: Zusammenführung und Vernet- } \\
\text { zung der Untersuchungsblogs verschiede- } \\
\text { ner Gruppen in Diskussion und Evaluation } \\
\text { des Themas }\end{array}$ \\
\hline $\begin{array}{l}\text { 3.1.2 «Eine Produktion planen und in ver- } \\
\text { schiedenen Formaten gestalten, prä- } \\
\text { sentieren, veröffentlichen oder teilen» }\end{array}$ & $\begin{aligned} \rightarrow & \text { Phase III bis VI: Planung (teilweise durch } \\
& \text { Lehrkraft), Gestaltung und Veröffentli- } \\
& \text { chung des Untersuchungsblog } \\
\rightarrow & \text { Phase IV und V: Formatvariabilität durch } \\
& \text { Variabilität der Datenaufnahme und Daten- } \\
& \text { verarbeitung in verschiedenen Repräsenta- } \\
& \text { tionsformen (z.B. Gleichungen, Graphen) }\end{aligned}$ \\
\hline
\end{tabular}


Kompetenzbereich: Problemlösen und Handeln

\begin{tabular}{|c|c|c|}
\hline \multicolumn{2}{|c|}{ Teilkompetenz } & \multirow[b]{2}{*}{$\begin{array}{l}\text { Implementierung in FELS } \\
\rightarrow \text { Phase III: Planung der Verwendung digita- } \\
\text { ler Werkzeuge beim Experimentieren und } \\
\text { Auswerten } \\
\rightarrow \text { Phase IV und V: Durchführung und Auswer- } \\
\text { tung der Experimente }\end{array}$} \\
\hline 5.2 .1 & $\begin{array}{l}\text { «Eine Vielzahl von digitalen Werkzeu- } \\
\text { gen kennen und kreativ anwenden» }\end{array}$ & \\
\hline 5.2 .2 & $\begin{array}{l}\text { «Anforderungen an digitale Werkzeuge } \\
\text { formulieren» }\end{array}$ & $\begin{aligned} & \rightarrow \text { Phase III: Planung der Verwendung digita- } \\
& \text { ler Werkzeuge beim Experimentieren und } \\
& \text { Auswerten } \\
& \rightarrow \text { Phase V: Begründung des Vorgehens bei } \\
& \text { der Untersuchung und Erstellung helfen- } \\
& \text { der Kommentare für andere Gruppen } \\
& \rightarrow \text { Phase VI: Darlegung der Überlegungen in } \\
& \text { der Präsentation }\end{aligned}$ \\
\hline 5.2 .3 & $\begin{array}{l}\text { «Passende Werkzeuge zur Lösung iden- } \\
\text { tifizieren» }\end{array}$ & $\rightarrow$ Phase II: je nach Kenntnisstand \\
\hline 5.4 .2 & $\begin{array}{l}\text { «Persönliches System von vernetzten } \\
\text { digitalen Lernressourcen selbst organi- } \\
\text { sieren können» }\end{array}$ & $\begin{aligned} \rightarrow & \text { Phase IV - VI: Bedienung verschiedener } \\
& \text { digitaler Werkzeuge auf unterschiedlichen } \\
& \text { Endgeräten } \\
\rightarrow & \text { Phase V: Transferierung der Daten in den } \\
& \text { Untersuchungsblog } \\
\rightarrow & \text { Phase IV und V: Selbstorganisation des } \\
& \text { Netzwerks bedingt durch ausserunterricht- } \\
& \text { liche Lernumgebung }\end{aligned}$ \\
\hline 5.5 .1 & $\begin{array}{l}\text { «Funktionsweisen und grundlegende } \\
\text { Prinzipien der digitalen Welt kennen } \\
\text { und verstehen.» }\end{array}$ & $\begin{aligned} \rightarrow & \text { in Abhängigkeit von Kenntnisstand der SuS } \\
& \text { und Zielen der Lehrkraft: } \\
\rightarrow & \text { Phase II: Kennen und Verstehen von zu } \\
& \text { nutzenden Sensoren und Apps } \\
\rightarrow & \text { Phase III: siehe oben; implizit hilfreich bei } \\
& \text { Erstellung des Untersuchungsplans }\end{aligned}$ \\
\hline 5.5 .3 & $\begin{array}{l}\text { «Eine strukturierte, algorithmische } \\
\text { Sequenz zur Lösung eines Problems } \\
\text { planen und verwenden» }\end{array}$ & $\begin{aligned} & \rightarrow \text { implizit: Betrachtung der Vorgehensweise } \\
& \text { bei FELS (Experimentierkreislauf) als struk- } \\
& \text { turierter Algorithmus zur Problemlösung }\end{aligned}$ \\
\hline
\end{tabular}

Kompetenzbereich «Analysieren und Reflektieren»

\begin{tabular}{|l|l|}
\hline Teilkompetenz & Implementierung in FELS \\
\hline 6.2.6 «Potenziale der Digitalisierung im & $\rightarrow$ Phase V und VI (implizit): Tipps als Kom- \\
$\begin{array}{l}\text { Sinne sozialer Integration und sozialer } \\
\text { Teilhabe erkennen, analysieren und } \\
\text { reflektieren» }\end{array}$ & $\begin{array}{l}\text { mentare im Untersuchungsblog können } \\
\text { Qualität der Untersuchungen und Präsen- } \\
\text { tationsleistung ( } \rightarrow \text { Unterrichtsqualität) er- } \\
\text { höhen; Bewusstmachung obliegt Lehrkraft }\end{array}$ \\
\hline
\end{tabular}




\section{Ausblick}

FELS als smartphonegestützte Lernumgebung zum Experimentieren stellt eine mögliche Umsetzung der KMK-Richtlinien zur Medienbildung für den Physikunterricht im Speziellen bzw. naturwissenschaftlichen Unterricht im Allgemeinen vor. Obgleich es zu untersuchen gilt, inwiefern FELS den oben formulierten Ansprüchen und Zielen gerecht wird, d.h. inwiefern mit FELS physikbezogene Kompetenzen sowie Medienkompetenzen ausgebildet, affektive Aspekte gefördert und damit die Einstellung zum Physikunterricht verbessert werden. Im praktischen Schuleinsatz wird es durch die genutzten Beispiele zu einer entsprechenden Spezifizierung und damit auch verbundenen Reduzierung der einzelnen Lernziele kommen. Hierdurch spannen die oben formulierten Forschungsvorhaben quasi einen eigenen kleinen Forschungsbereich auf, den es künftig zu bearbeiten gilt.

Urahne et al. (2000) forderte bereits vor über 10 Jahren, dass Didaktiker stärker in die Softwareentwicklung bezüglich Lernprogramme etc. eingebunden werden sollten. Zur besseren Umsetzung der oben skizzierten Forschung wird an der Universität Potsdam eine Android-App entwickelt, die die verschiedenen Phasen von FELS abdeckt. Bislang ist der Zyklus abgedeckt und gängige Sensoren sind in die App eingebunden. Dabei können neben physikalischen Messungen auch Texte geschrieben und Bilder aufgenommen werden, die zunächst auf dem Gerät gespeichert und dann per email versendet oder per soziale Netzwerke geteilt werden können. Später sollen diese jedoch automatisch in den Onlineblog hochgeladen werden. Ausserdem sollen weitere Messmodi ergänzt werden, um die Einsatzbarkeit zu erweitern. Danach soll FELS als methodische, übergeordnete Rahmenstruktur für PU mit weiteren Praxisbeispielen ausgebaut werden.

\section{Literatur}

Bachmair, Ben, John Coole, und Norbert Pachler. 2014. «Mobile Medien als Kulturressourcen für Lernen, ein kulturökologischer Beitrag zur Medienbildung». In: Perspektiven der Medienbildung, hrsg. v. Winfried Marotzki und Norbert Meder. 209-233. Wiesbaden: Springer.

Banchi, Heather, und Randy Bell. 2008. «The many levels of inquiry». Science and Children (Oktober 2008): 26-29.

Barth, Johannes Maximilian. 2014. Experimentieren im Physikunterricht der gymnasialen Oberstufe. Eine Rekonstruktionübergeordneter Einbettungsstrategien. Berlin: Logos-Verlag.

Becker, Sebastian, Pascal Klein, Alexander Gößling, und Jochen Kuhn. 2017. «Technologie-unterstütztes Lernen im Physikunterricht mittels mobilder Videoanalyse». Lernprozesse mit digitalen Werkzeugen unterstützen. Perspektiven aus der Didaktik naturwissenschaftlicher Fächer, hrsg. v. Jenny Meßinger-Koppelt, Sascha Schanze und Jorge Groß. 119-130. Hamburg: Joachim Herz Stiftung. 
Börlin, Johannes. 2012. Das Experiment als Lerngelegenheit. Vom interkulturellen Vergleich des Physikunterrichts zu Merkmalen seiner Qualität. Berlin: Logos.

Cabeza, Cecilia, Nicolás Rubido, und Arturo C. Martí. 2014. «Learning physics in a water park» PHYSICS EDUCATION 49 (2): 187-194.

Castro-Palacio, Juan Carlos, Luisberis Velázquez-Abed, Marcos H. Giménez, und Juan A. Monsoriu. 2013. «Using a mobile phone acceleration sensor in physics experiments on free and damped harmonic oscillations». Am. J. Phys. 81 (6), 472-475. https://doi. org/10.1119/1.4793438.

de Witt, Claudia, und Thomas Czerwionka. 2007. Mediendidaktik. Studientexte für Erwachsenenbildung. Bielefeld: Bertelsmann Verlag.

de Witt, Claudia. 2013. «Vom E-Learning zum Mobile Learning - wie Smartphones und Tablet PCs Lernen und Arbeiten verbinden». In: Mobile Learning. Potenziale, Einsatzszenarien und Perspektiven des Lernens mit mobilen Endgeräten, hrsg. v. Claudia de Witt und Almut Reiners, 55-74, Wiesbaden: Springer.

Duit, Reinders, Peter Häussler, und Ernst Kircher. 1981. Unterricht Physik. Materialien zur Unterrichtsvorbereitung. Köln: Aulis.

Edelson, Daniel C., Douglas N. Gordin, und Roy D. Pea. 1999. «Adressing the Challenges of Inquiry-Based Learning Through Technology and Curriculum Design». THE JOURNAL OF THE LEARNING SCIENCES, 3\&4(8): 391-450. https://hal.archives-ouvertes.fr/hal-00190609/ document.

Emden, Markus, und Elke Sumfleth. 2012. «Prozessorientierte Leistungsbewertung des experimentellen Arbeitens - Zur Eignung einer Protokollmethode zur Bewertung von Experimentierprozessen». MNU 2(65): 68-75.

Engl, Alexander, und Björn Risch. 2015. «Chemie Pur - Unterrichten in der Natur». In: Heterogenität und Diversität - Vielfalt der Voraussetzungen im naturwissenschaftlichen Unterricht, hrsg. v. Sascha Bernholt. 546-548. Kiel: IPN-Verlag.

Feierabend, Sabine, Theresa Plankenhorn, und Thomas Rathgeb. 2015. «JIM 2015. Jugend, Information, (Multi-) Media. Basisstudie zum Medienumgang 12- bis 19-Jähriger in Deutschland». hrsg. v. Medienpädagogischer Forschungsverbund Südwest. http://mpfs.de/fileadmin/JIM-pdf15/JIM_2015.pdf.

Feierabend, Sabine, Theresa Plankenhorn, und Thomas Rathgeb. 2016. «JIM 2016. Jugend, Information, (Multi-) Media. Basisstudie zum Medienumgang 12- bis 19-Jähriger in Deutschland». hrsg. v. Medienpädagogischer Forschungsverbund Südwest. https://www.mpfs.de/ fileadmin/files/Studien/JIM/2016/JIM_Studie_2016.pdf.

Franke-Braun, Gudrun, Florian Schmidt-Weigand, Lutz Stäudel, und Rita Wodzinski. 2008. «Aufgaben mit gestuften Lernhilfen - ein besonderes Aufgabenformat zur kognitiven Aktivierung der Schülerinnen und Schüler und zur Intensivierung der sachbezogenen Kommunikation». In: Lernumgebungen auf dem Prüfstand. Zwischenergebnisse aus den Forschungsprojekten, hrsg. v. Kasseler Forschergruppe. 27-42. Kassel: kassel university press GmbH. http://www.uni-kassel.de/upress/online/frei/978-3-89958-394-6.volltext.frei.pdf. 
Frischknecht-Tobler, Ursula, und Peter Labudde. 2010. «Beobachten und Experimentieren». In: Fachdidaktik Naturwissenschaft: 1. - 9. Schuljahr, hrsg. v. Peter Labudde. 133-148. Bern: Haupt.

Gesellschaft für Medienpädagogik und Kommunikationskultur. 2018. Medienbildung in der Schule ist mehr als digitale bildung - Medienpädagogik positioniert sich zur Strategie der Kultusministerkonferenz 'Bildung in der digitalen Welt'. https://www.gmk-net.de/wp-content/uploads/2018/09/gmk-positionspapier_kmk-strategie_27092018.pdf.

Grell, Petra, und Franco Rau. 2011. «Partizipationslücken - Social Software in der Hochschullehre». Zeitschrift MedienPädagogik 21, https://doi.org/10.21240/mpaed/21/2011.11.21.X.

Hagelgans, Heike. 2015. «Der ganzheitliche Anstoß - wie eine Billardlernwerkstatt die Potenzialentfaltung von Schülerinnen und Schülern und die Unterrichts- und Schulentwicklung anregen kann - Ein- und Ausblicke in eine wissenschaftliche Begleitstudie». Schulpädagogik heute 11(6). 16.

Hmelo-Silver, Cindy E., Ravit Golan Duncan, und Clark A. Chinn. 2007. «Scaffolding and Achievement in Problem-Based and Inquiry Learning: A Response to Kirschner, Sweller, and Clark (2006)». EDUCATIONAL PSYCHOLOGIST 2(42). 99-107.

Hofferber, Natalia, Alexander Eckes, Anastassiya Kovaleva, und Matthias Wilde. 2015. «Die Auswirkungen von autonomieförderndem Lehrerverhalten im Biologieunterricht mit lebenden Tieren». ZfDN 21. 17-27.

Hoffmann, Lore, Peter Häußler, und Manfred Lehrke. 1998. Die IPN Interessensstudie. Kiel:IPN

Höttecke, Dietmar, und Falk Rieß. 2015. «Naturwissenschaftliches Experimentieren im Lichte der jüngeren Wissenschaftsforschung - Auf der Suche nach einem authentischen Experimentbegriff der Fachdidaktik». ZfDN 21. 127-139. https://doi.org/10.1007/s40573-0150030-z.

Horvath, Patrick. 2007. «Was tun mit den «digital natives»? Herausforderungen für Wirtschaft, Politik und Bildungssystem jenseits bloßer Bewährungspädagogik». medienimpulse 60 . 34-36. http://www2.mediamanual.at/themen/kompetenz/60_Horvath-Was_tun_mit.pdf.

Hucke, Lorenz. 1999. Handlungsregulation und Wissenserwerb in traditionellen und computergestützten Experimenten des physikalischen Praktikums. Berlin: Logos.

Huwer, Johannes, und Ingo Eilks. 2017. «Multitouch Learning Books für schulische und außerschulische Bildung». In Lernprozesse mit digitalen Werkzeugen unterstützen. Perspektiven aus der Didaktik naturwissenschaftlicher Fächer, hrsg. v. Jenny Meßinger-Koppelt, Sascha Schanze und Jorge Groß. 81-94. Hamburg: Joachim Herz Stiftung.

Jonas-Ahrend, Gabriela. 2004. Physiklehrervorstellungen zum Experiment im Physikunterricht. Berlin: Logos.

Kohler, Britta. 2011. "Hausaufgaben. Überblick über didaktische Überlegungen und empirische Untersuchungen». DDS (3)103. 203-218.

Kruse, Ronald. 2014. «GPS im Physikunterricht - Analyse mechanischer Bewegungen mit Hilfe eines Smartphones». In Digitale Medien im naturwissenschaftlichen Unterricht, hrsg. v. Jörg Maxton-Küchenmeister und Jenny Meßinger-Koppelt. 162-166. Hamburg: Joachim Herz Stiftung. 
Kuhn, Jochen. 2010. Authentische Aufgaben im theoretischen Rahmen von Instruktions- und Lehr-Lern-Forschung: Effektivität und Optimierung von Ankermedien für eine neue Aufgabenkultur im Physikunterricht. Wiesbaden: Vieweg+Teubner Verlag.

Kuhn, Jochen. 2011. «Zeitungsaufgaben und Co.: Einsatz und Effektivität authentischer Lernmedien im Physikunterricht». Plus Lucis 1-2. 11-17. https://pluslucis.univie.ac.at/PlusLucis/111/S11.pdf.

Kuhn, Jochen, Patrick Vogt, und Sebastian Müller. 2011. «Handys und Smartphones. Einsatzmöglichkeiten und Beispielexperimente im Physikunterricht». PdN PHYSIK in der Schule 7(60). 5-11.

Kuhn, Jochen, und Patrick Vogt. 2013. «Applications and Examples of Experiments with Mobile Phones and Smartphones in Physics Lessons». Frontiers in Sensors 1(4). 67-73. http://www. seipub.org/fs/Download.aspx?ID=9682.

Kuhn, Jochen, Patrick Vogt, und Florian Theilmann. 2016. «Going nuts. Measuring free-fall acceleration by analyzing the sound of falling metal pieces». The Physics Teacher 54(3). 182183. https://doi.org/10.1119/1.4942145.

Labudde, Peter, und Johannes Börlin. 2013. «Inquiry-Based-Learning: Versuch einer Einordnung zwischen Bildungsstandarts, Forschungsfeldern und PROFILES». In Inquiry-based Learning - Forschendes Lernen, hrsg. v. Sascha Bernholt. 183-185. Kiel: IPN-Verlag.

Laukenmann, Mathias, Michael Bleicher, Stefan Fuß, Michaela Gläser-Zikuda, Philipp Mayring, und Christoph von Rhöneck. 2000. «Eine Untersuchung zum Einfluss emotionaler Faktoren auf das Lernen im Physikunterricht». ZfDN 6. 134-155. ftp://ftp.rz.uni-kiel.de/pub/ipn/ zfdn/2000/S.139-155_Laukenmann_etal._2000.pdf.

Leisen, Josef, Hrsg. 1999. Methodenhandbuch deutschsprachiger Fachunterricht DFU. Bonn: Varus.

Lück, Stephan, und Thomas Wilhelm. 2011. «Beschleunigungspfeile mit dem iPhone». PdN PHYSIK in der Schule 7(60). 27-29.

Merzyn, Gottfried. 2015. «Guter Physikunterricht. Die Sicht von Schülern, Lehrern und Wissenschaftlern». phydid B - Beiträge zur DPG-Frühjahrstagung, 8. http://www.phydid.de/index. php/phydid-b/article/view/589/745.

Mikelskis-Seifert, Silke, und Reinders Duit. 2010. Physik im Kontext - Konzepte, Ideen, Materialien für effizienten Physikunterricht. Seelze: Friedrich Verlag.

Ministerium für Bildung, Jugend und Sport. 2015. «Rahmenlehrplan Teil C Physik Jahrgangsstufen 7-10». https://bildungsserver.berlin-brandenburg.de/fileadmin/bbb/unterricht/rahmenlehrplaene/Rahmenlehrplanprojekt/amtliche_Fassung/Teil_C_Physik_2015_11_16_ web.pdf.

Müller, Jirka, Uta Magdans, und Andreas Borowski. 2016. «Der Einsatz von Smartphones und Tablet-PCs in Physiklehramts- und Schüler_innenpraktika». In Lehrende arbeiten mit dem Netz, hrsg. v. Meral Akin-Hecke, Monika Andraschko, Peter Eiselmair, und David. S. Röthler, 228-231. Wien: Werde Digital.at \& Education Group.

Müller, Jirka, Robert Scharner, und Oliver Henneberg. 2014. «LabVIEW im Informatik- und Physikunterricht». PdN Physik in der Schule 5(63). 36-39. 
Nieswand, Martina. 2014. Hausaufgaben yapmak. Ein ethnographischer Blick auf den Familienalltag. Bad Heilbrunn: Klinkhardt.

Oberländer, Arne. 2014. «Schulkontext - Lebenswelt und Unterricht mobil verbinden». In Digitale Medien im Naturwissenschaftlichen Unterricht, hrsg. v. Jörg Maxton-Küchenmeister und Jenny Meßinger-Koppelt. 271-279, Hamburg: Joachim Herz Stiftung Verlag.

O'Reilly, Tim. 2006. «Web 2.0 Compact Definition: Trying Again». http://radar.oreilly. com/2006/12/web-20-compact-definition-tryi.html.

Rabe, Thorid, und Helmut F. Mikelskis. 2007. «Kohärenzbildungshilfen und Selbsterklärungen: Fördern sie das Physiklernen?». ZfDN 13. 33-52. http://archiv.ipn.uni-kiel.de/zfdn/ pdf/002Rabe_13.pdf.

Rath, Gerhard, und Eduard Schittelkopf. 2011. «Mobile@classroom Handyclips im Physikunterricht» PdN PHYSIK in der Schule 7(60). 12-14.

Reitinger, Johannes. 2012. «Differenziertes forschendes Lernen in den Naturwissenschaften mit leistungsheterogenen Schüler/-innengruppen. Eine empirische Studie zur Performanz und Wirksamkeit des AuRELIA-Konzeptes». In Binnendifferenzierung, Didaktische Grundlagen und Forschungsergebnisse zur Binnendifferenzierung im Unterricht Teil l, hrsg. v. Thorsten Bohl, Manfred Bönsch, Matthias Trautmann und Beate Wischer. 107-133, Immenhausen bei Kassel: Prolog-Verlag.

Reitinger, Johannes. 2014. Forschendes Lernen. Theorie, Evaluation und Praxis in naturwissenschaftlichen Lernarrangements. Immenhausen bei Kassel: Prolog-Verlag, 2. Auflage.

Sander, Florian. 2000. Verbindung von Theorie und Experiment im physikalischen Praktikum. Eine empirische Untersuchung zum handlungsbezogenen Vorverständnis und dem Einsatz grafikorientierter Modellbildung im Praktikum. Berlin: Logo.

Schacter, Daniel L. 1996. Searching for memory. The brain, the mind, and the past. New York: Basic books.

Schmidtkunz, Heinz, und Helmut Lindemann. 1999. Das forschend-entwickelnde Unterrichtsverfahren. Problemlösen im naturwissenschaftlichen Unterricht. Magdeburg: Westarp Wissenschaften, 5. Auflage.

Schwab, Joseph J. 1960. «Inquiry, the Science Teacher, and the Educator». The school review 2(68). 176-195.

Schwemmer, Hilmar. 1980. Was Hausaufgaben anrichten: Von der Fragwürdigkeit eines Jahrhunderte verewigten Tabus in der Hausaufgabenschule unserer Zeit. Paderborn: Schöningh.

Sekretariat der Ständigen Konferenz der Kultusminister der Länder in der Bundesrepublik Deutschland, Hrsg .2005. Strategie der Kultusministerkonferenz. Bildungsstandards im Fach Physik für den mittleren Schulabschluss. Beschluss vom 16.12.2004. http://www.kmk.org/ fileadmin/Dateien/veroeffentlichungen_beschluesse/2004/2004_12_16-Bildungsstandards-Physik-Mittleren-SA.pdf

Sekretariat der Ständigen Konferenz der Kultusminister der Länder in der Bundesrepublik Deutschland, Hrsg. 2016. Beschlüsse der Kultusministerkonferenz. Bildung in der digitalen Welt. Beschluss vom 08.12.2016. https://www.kmk.org/fileadmin/Dateien/pdf/PresseUndAktuelles/2016/Bildung_digitale_Welt_Webversion.pdf 
Smeds, Pia, Eila Jeronen, und Sirpa Kurppa. 2015. «Farm Education and the Value of Learning in an Authentic Learning Environment». International Journal of Environmental \& Science Education 10(3). 381-404. https://doi.org/10.12973/ijese.2015.251a.

Spang, André. 2014. «Mobiles Lernen mit Tablets, Apps und cloud - Ein Erfahrungsbericht». In Digitale Medien im naturwissenschaftlichen Unterricht, hrsg. v. Jörg Maxton-Küchenmeister und Jenny Meßinger-Koppelt. 101-113. Hamburg: Joachim Herz Stiftung.

Specht, Marcus, Marco Kalz, und Dirk Börner. 2013. «Innovation und Trends für Mobiles Lernen». In Mobile Learning. Potenziale, Einsatzszenarien und Perspektiven des Lernens mit mobilen Endgeräten, hrsg. v. Claudia de Witt und Almut Reiners, 55-74, Wiesbaden: Springer.

Staacks, Sebastian. 2018. «Phyphox. Unterstützte Sensoren». https://phyphox.org/de/unterstutzte-sensoren/.

Streller, Sabine. 2013. «PROFILES in der Lehramtsausbildung». In Inquiry-based Learning - Forschendes Lernen, hrsg. v. Sascha Bernholt. 194 - 196. Kiel: IPN-Verlag.

Sumfleth, Elke, und Nina Nicolai. 2009. «Kooperative Hausaufgaben im Chemieunterricht. Ergebnisse einer Videostudie». MNU 1(62). 46-54.

Sumfleth, Elke, Cornna Kieren, und Isabell van Ackeren. 2011. "Hausaufgabenpraxis im Gymnasium - Empirische Befunde am Beispiel eines 'Nebenfachs'». DDS 3(103). 252-267. https://www.dds.uni-hannover.de/fileadmin/schulentwicklungsforschung/DDS_Open_Access/DDS_11_3_Hausaufgaben_Lerngelegenheiten.pdf.

Tesch, Maike. 2005. Das Experiment im Physikunterricht. Didaktische Konzepte und Ergebnisse einer Videostudie. Berlin: Logos.

Thyssen, Christoph. 2017. «Augmented Reality (AR) im praktischen Unterricht». In Lernprozesse mit digitalen Werkzeugen unterstützen. Perspektiven aus der Didaktik naturwissenschaftlicher Fächer, hrsg. v. Jenny Meßinger-Koppelt, Sascha Schanze und Jorge Groß. 177-191. Hamburg: Joachim Herz Stiftung.

Urahne, Detlef, Manfred Prenzel, Matthias von Davier, Martin Senkbeil, und Michael Bleschke. 2000. «Computereinsatz im naturwissenschaftlichen Unterricht - Ein Überblick über die pädagogisch-psychologischen Grundlagen und ihre Anwendung». ZfDN 6. 157-186. ftp:// ftp.rz.uni-kiel.de/pub/ipn/zfdn/2000/S.157-186_Urhahne_Prenzel_etal_2000.pdf.

van Vorst, Helena, Alexandra Vivien Dorschu, Sabine Fechner, Alexander Kauertz, Heiko Krabbe, und Elke Sumfleth. 2014. "Charakterisierung und Strukturierung von Kontexten im naturwissenschaftlichen Unterricht - Vorschlag einer theoretischen Modellierung». ZfDN 21. 29-39. https://doi.org/10.1007/s40573-014-0021-5.

Vogt, Patrick, Lutz Kasper, und Andreas Müller. 2014. «Smartphone Physics: Neue Experimente und Fragestellungen rund um das Messwerterfassungssystem Smartphone», phydid B Beiträge zur DPG-Frühjahrstagung, 12. http://www.phydid.de/index.php/phydid-b/article/ view/546/.

Vogt, Patrick, Jochen Kuhn, und Simon Gareis. 2011. «Beschleunigungssensoren von Smartphones Beispielexperimente zum Einsatz im Physikunterricht». PdN PHYSIK in der Schule $7(60) .15-22$. 
Wahser, Isabel, und Elke Sumfleth. 2008. «Training experimenteller Arbeitsweisen zur Unterstützung kooperativer Kleingruppen im Fach Chemie». ZfDN 14. 219-241. http://archiv.ipn. uni-kiel.de/zfdn/pdf/14_012_Wahser_Sumfleth.pdf.

West, Mark, und Steven Vosloo. 2013. UNESCO policy guidelines for mobile learning. Paris: UNESCO Publications. http://unesdoc.unesco.org/images/0021/002196/219641e.pdf.

Widodo, Ari, und Reinders Duit. 2005. «Konstruktivistische Lehr-Lern-Sequenzen und die Praxis des Physikunterrichts». ZfDN 11. 131-146. ftp://ftp.rz.uni-kiel.de/pub/ipn/zfdn/2005/9. Widodo_Duit_131-146.pdf.

Winkelmann, Jan. 2015. Auswirkungen auf den Fachwissenszuwachs und auf affektive Schülermerkmale durch die Schüler- und Demonstrationsexperimente im Physikunterricht. Berlin: Logos.

Wodzinski, Rita. 2013. «Lernen mit gestuften Hilfen». Physik Journal 12. 45-49.

Zumbach, Jörg, Stephanie Mooser, Ulrike Unterbauer, und Georg Pfligersdorffer. 2014. «Problemorientiertes Online-Lernen im Biologieunterricht: Fähigkeitsselbstkonzept, mentale Anstrengung und Vorwissen als Prädiktoren für Wissenserwerbsprozesse zwischen Instruktion und Konstruktion». ZfDN 20. 45-56. https://doi.org/10.1007/s40573-014-0004-6. 